\title{
A comparative study of cervical hysteresis characteristics after various osteopathic manipulative treatment (OMT) modalities.
}

\author{
Barnes PL, Laboy F, Noto-Bell L, Nelson J, Ferencz V, Nicholas AS, Kuchera ML. \\ Human Performance \& Biomechanics Laboratory of the Department of Osteopathic Manipulative Medicine and Center for \\ Chronic Disorders of Aging; Philadelphia College of Osteopathic Medicine, Philadelphia, PA, USA.
}

\begin{abstract}
BACKGROUND: Despite apparent clinical benefits, few objective tissue texture measurements exist documenting post-OMT change. Spineliner ${ }^{\circledR}$ technology is capable of measuring hysteresis (tissue texture) changes adjacent to the spine before and after treatment interventions. The technology could be employed to document changes before and after OMT and to determine if varied outcomes result using different OMT activating forces in the cervical spine.
\end{abstract}

METHODS: 200 subjects were equally and randomly assigned to receive Sham or single-segmental Muscle Energy (ME), Counterstrain (CS), Balanced Ligamentous Tension (BLT), or High-Velocity Low-Amplitude (HVLA) OMT. After palpatory diagnosis for somatic dysfunction, subjects were objectively measured (Spineliner ${ }^{\circledR}$ ), treated with cervical OMT, and then remeasured (Spineliner ${ }^{\circledR}$ ). Of four components used to calculate a durometer, motoricity (area under the curve) and fixation (tissue resistance) were analyzed before and after OMT for each level and for each activating force

RESULTS: Statistically significant or highly suggestive changes in motoricity (OA, C2-C5) and fixation (OA, C3, C5-6) were generally seen post-OMT. Regardless of treatment type, the most significant changes in fixation and motoricity occurred at C5. There was an overall trend suggesting that of these procedures, ME provides the greatest immediate improvement of hysteresis characteristics. While fixation immediately increased at C2 when using BLT, HVLA, or CS (in worsening order), it showed immediate improvement with ME. A suggestive motoricity trend was also observed at the level of the OA, inferring an improved treatment response was obtained with BLT, HVLA, CS, and ME (least to most responsive order).

CONCLUSIONS: Comparing treated to untreated cervical spines, nearly all levels demonstrated immediate objective hysteresis change post-OMT. C5 showed the most change regardless of treatment type; ME provided the greatest immediate change. 
